\title{
de Sitter relativity in static charts
}

\author{
Ion I. Cotăescu ${ }^{\text {a }}$ \\ West University of Timişoara, V. Pârvan Ave. 4, 300223 Timisoara, Romania
}

Received: 20 August 2017 / Accepted: 24 January 2018 / Published online: 1 February 2018

(C) The Author(s) 2018. This article is an open access publication

\begin{abstract}
The relative geodesic motion in static (and spherically symmetric) local charts on the $(1+3)$-dimensional de Sitter spacetimes is studied in terms of conserved quantities. The Lorentzian isometries are derived, relating the coordinates of the local chart of a fixed observer with the coordinates of a mobile chart considered as the rest frame of a massive particle freely moving on a timelike geodesic. The time dilation and Lorentz contraction are discussed pointing out some notable features of the de Sitter relativity in static charts.
\end{abstract}

\section{Introduction}

The simplest $(1+3)$-dimensional spacetimes of special or general relativity are vacuum solutions of the Einstein equations whose geometry is determined only by the value of the cosmological constant $\Lambda$. These are the Minkowski flat spacetime (with $\Lambda=0$ ), and the hyperbolic spacetimes, de Sitter (dS) with $\Lambda>0$ and Anti-de Sitter (AdS) having $\Lambda<0$ [1]. All these spacetimes have highest possible isometries [2] representing thus a good framework for studying the role of the conserved quantities with physical meaning in quantum theory [3-6] or for describing the classical relative geodesic motion [7-9]. With their help we constructed recently the dS relativity [10] in comoving charts [11] and the AdS relativity [12] in static and spherically symmetric local charts that complete our image of the special relativity in spacetimes with maximal symmetry.

Our approach is based on the idea that the inertial (natural) frames are local charts playing the role of rest frames of massive particles freely moving along timelike geodesics. Moreover, we impose a synchronization condition requiring the origins of the fixed and moving frames to overlap at a given time. The conserved quantities on these geodesics help us to mark the different inertial frames whose relative motion can then be studied by using the Nachtmann boosting method

\footnotetext{
a e-mail: i.cotaescu@e-uvt.ro
}

of introducing coordinates in different dS local charts [3]. In this manner, we derived the Lorentzian isometries relating the coordinates of the moving and fixed inertial frames on dS or AdS backgrounds $[10,12]$.

The $(1+3)$-dimensional AdS spacetime is the only maximally symmetric spacetime which does not have space translations [2], since its $\Lambda<0$ produces an attraction of elastic type such that the geodesic motion is oscillatory around the origins of the static charts with ellipsoidal closed trajectories. The AdS relativity relates these charts such that, according to the synchronization condition, the moving frames may have only rectilinear geodesics whose oscillatory motions are centered in the origin of the fixed frame [12]. On the contrary, in the comoving local charts we used so far (i.e. the conformal Euclidean and de Sitter-Painlevé ones), the dS relativity seems to be closer to the Einstein special relativity since here we have translations and conserved momenta such that at least in the conformal Euclidean chart all the geodesic trajectories are rectilinear along the momentum direction $[6,10]$.

However, apart from the comoving charts, the dS spacetime has, in addition, static charts where the geodesic trajectories are no longer rectilinear such that the role of the conserved momentum becomes somewhat obscure. Since the dS relativity in these charts is not yet formulated, we focus here on this problem studying the role of the conserved quantities along geodesics in describing the relative geodesic motion.

In order to preserve the coherence of our dS relativity, we use here the same definitions, conventions and initial conditions as in Ref. [10] since then we can take over the results obtained therein without revisiting the entire boosting method which allowed us to construct the dS and AdS relativity. In this manner we obtain a version of the dS relativity in static charts which is perfectly symmetric with the AdS one with respect to the change of the hyperbolic functions into trigonometric ones.

The principal new result we report here concern the role of the conserved quantities in determining the parametrization of the Lorentzian isometries relating fixed and moving static 
charts. Moreover, we briefly discuss some notable properties of these isometries and their consequences upon simple relativistic effects as the time dilation and Lorentz contraction.

We start in the second section with a short review of the static charts where we consider the dS conserved quantities presented in the third section. The next section is devoted to the timelike geodesics in static charts showing how their integration constants depend on the conserved quantities with physical meaning and pointing out the kinematic role of these quantities. In Sect. 5 we solve the relativity problem in static charts deriving the Lorentzian isometries with different parametrizations. In the last part of this section we discuss the above-mentioned simple relativistic effects, giving the general formulas, allowing the analytical and numerical study of particular cases. In the last section we present the dS-AdS symmetry which involves all the conserved quantities of both these spacetimes.

\section{Static charts on dS spacetimes}

Let us consider the $(1+3)$-dimensional dS spacetime $(M, g)$ which is a vacuum solution of the Einstein equations with $\Lambda>0$ and positive constant curvature. This is a hyperboloid of radius $R=\frac{1}{\omega}=\sqrt{\frac{3}{\Lambda}}$ embedded in the $(1+4)$ dimensional pseudo-Euclidean spacetime $\left(M^{5}, \eta^{5}\right)$ of Cartesian coordinates $z^{A}$ (labeled by the indices $A, B, \ldots=$ $0,1,2,3,4)$ and metric $\eta^{5}=\operatorname{diag}(1,-1,-1,-1,-1)$. These coordinates are global, corresponding to the pseudoorthonormal basis $\left\{v_{A}\right\}$ of the frame into consideration, whose unit vectors satisfy $v_{A} \cdot v_{B}=\eta_{A B}^{5}$. Any point $z \in M^{5}$ is represented by the five-dimensional vector $z=v_{A} z^{A}=$ $\left(z^{0}, z^{1}, z^{2}, z^{3}, z^{4}\right)^{T}$, which transforms linearly under the gauge group $S O(1,4)$ which leave the metric $\eta^{5}$ invariant.

The local static charts $\{x\}$, of coordinates $x^{\mu}(\alpha, . . \mu$, $v \cdots=0,1,2,3)$, can be introduced on $(M, g)$ giving the set of functions $z^{A}(x)$ which solve the hyperboloid equation,

$\eta_{A B}^{5} z^{A}(x) z^{B}(x)=-\frac{1}{\omega^{2}}$.

The usual static chart $\{t, \mathbf{x}\}$ with Cartesian spaces coordinates $x^{i}(i, j, k, \ldots=1,2,3)$ is defined by

$z^{0}(x)=\frac{1}{\omega} \chi(r) \sinh (\omega t)$,

$z^{i}(x)=x^{i}$,

$z^{4}(x)=\frac{1}{\omega} \chi(r) \cosh (\omega t)$,

where $r=|\mathbf{x}| \leq \frac{1}{\omega}$ and $\chi(r)=\sqrt{1-\omega \mathbf{x}^{2}}=\sqrt{1-\omega^{2} r^{2}}$. Hereby one obtains the line element,

$$
\begin{aligned}
d s^{2} & =\eta_{A B}^{5} d z^{A}(x) d z^{B}(x) \\
& =\chi(r)^{2} d t^{2}-\left[\delta_{i j}+\omega^{2} \frac{x^{i} x^{j}}{\chi(r)^{2}}\right] d x^{i} d x^{j} .
\end{aligned}
$$

The associated static chart $\{t, r, \theta, \phi\}$ with spherical coordinates, canonically related to the Cartesian ones, $\mathbf{x} \rightarrow$ $(r, \theta, \phi)$, has the line element

$d s^{2}=\chi(r)^{2} d t^{2}-\frac{d r^{2}}{\chi(r)^{2}}-r^{2}\left(d \theta^{2}+\sin ^{2} \theta d \phi^{2}\right)$.

Apart from the above usual charts, it is useful to consider the static chart $\{\tilde{x}\}=\{t, \rho, \theta, \phi\}$ resulting after the substitution $[13,14]$,

$r=\frac{\rho}{\tilde{\chi}(\rho)}, \quad \tilde{\chi}(\rho)=\sqrt{1+\omega^{2} \rho^{2}}$,

where $\rho \in[0, \infty)$. Then the embedding equations become

$z^{0}(\tilde{x})=\frac{1}{\omega \tilde{\chi}(\rho)} \sinh (\omega t)$,

$z^{1}(\tilde{x})=\frac{\rho}{\tilde{\chi}(\rho)} \sin \theta \cos \phi$,

$z^{2}(\tilde{x})=\frac{\rho}{\tilde{\chi}(\rho)} \sin \theta \sin \phi$,

$z^{3}(\tilde{x})=\frac{\rho}{\tilde{\chi}(\rho)} \cos \theta$,

$z^{4}(\tilde{x})=\frac{1}{\omega \tilde{\chi}(\rho)} \cosh (\omega t)$,

while the line element reads

$d s^{2}=\frac{1}{\tilde{\chi}(\rho)^{2}}\left[d t^{2}-\frac{d \rho^{2}}{\tilde{\chi}(\rho)^{2}}-\rho^{2}\left(d \theta^{2}+\sin ^{2} \theta d \phi^{2}\right)\right]$.

In this chart the components of the four-velocity are denoted $\tilde{u}^{\mu}=\frac{d \tilde{x}^{\mu}}{d s}$.

\section{Conserved quantities}

The dS spacetimes are homogeneous spaces of the gauge group $S O(1,4)$ whose transformations leave invariant the metric $\eta^{5}$ of the embedding manifold $M^{5}$ and implicitly Eq. (1). For this group we adopt the canonical parametrization,

$\mathfrak{g}(\xi)=\exp \left(-\frac{i}{2} \xi^{A B} \mathfrak{S}_{A B}\right) \in S O(1,4)$

with skew-symmetric parameters, $\xi^{A B}=-\xi^{B A}$, and the covariant generators of the fundamental representation of the so $(1,4)$ algebra carried by $M^{5}$ having the matrix elements,

$\left(\mathfrak{S}_{A B}\right)_{\cdot D}^{C \cdot}=i\left(\delta_{A}^{C} \eta_{B D}^{5}-\delta_{B}^{C} \eta_{A D}^{5}\right)$. 
In any local chart $\{x\}$, defined by the functions $z=z(x)$, each transformation $\mathfrak{g} \in S O(1,4)$ gives rise to the associated isometry $x \rightarrow x^{\prime}=\phi_{\mathfrak{g}}(x)$ derived from the system of equations $z\left[\phi_{\mathfrak{g}}(x)\right]=\mathfrak{g} z(x)$.

The $s o(1,4)$ basis-generators with an obvious physical meaning [6-8] are the energy $\mathfrak{H}=\omega \mathfrak{S}_{04}$, angular momentum $\mathfrak{J}_{k}=\frac{1}{2} \varepsilon_{k i j} \mathfrak{S}_{i j}$, Lorentz boosts $\mathfrak{K}_{i}=\mathfrak{S}_{0 i}$, and the Runge-Lenz-type vector $\mathfrak{R}_{i}=\mathfrak{S}_{i 4}$. In addition, we consider the momentum $\mathfrak{P}_{i}=-\omega\left(\mathfrak{R}_{i}+\mathfrak{K}_{i}\right)$ and its dual $\mathfrak{Q}_{i}=\omega\left(\mathfrak{K}_{i}-\mathfrak{R}_{i}\right)$, which are nilpotent matrices of two Abelian three-dimensional subalgebras [6].

In general, after integrating the geodesic equations, one obtains the geodesic trajectories depending on some integration constants that must get a physical interpretation. This is possible only by expressing them in terms of conserved quantities on geodesics. These are given by the Killing vectors associated to the $S O(1,4)$ isometries which are defined (up to a multiplicative constant) as [4],

$\mathfrak{S}_{A B} \rightarrow k_{(A B) \mu}=z_{A} \partial_{\mu} z_{B}-z_{B} \partial_{\mu} z_{A}$,

where $z_{A}=\eta_{A C}^{5} z^{C}$. The principal conserved quantities along a timelike geodesic of a pointlike particle of mass $m$ and momentum $\mathbf{P}$ have the general form

$\mathscr{K}_{(A B)}(x, \mathbf{P})=\omega k_{(A B) \mu} m u^{\mu}$

where $u^{\mu}=\frac{d x^{\mu}(s)}{d s}$ are the components of the covariant four-velocity that satisfy $u^{2}=g_{\mu \nu} u^{\mu} u^{\nu}=1$. The conserved quantities with physical meaning $[6-8,10]$ are the well-known energy and angular momentum,

$$
\begin{aligned}
E & =\omega k_{(04) \mu} m u^{\mu} \\
L_{i} & =\frac{1}{2} \varepsilon_{i j k} k_{(j k) \mu} m u^{\mu}
\end{aligned}
$$

and the $S O(3)$ vectors having the components,

$K_{i}=k_{(0 i) \mu} m u^{\mu}$,

$R_{i}=k_{(i 4) \mu} m u^{\mu}$,

related to the conserved momentum, and $\mathbf{P}$ and its dual $\mathbf{Q}$ defined as [6],

$P^{i}=-\omega\left(R_{i}+K_{i}\right), \quad Q^{i}=\omega\left(K_{i}-R_{i}\right)$.

Thus we can construct the five-dimensional matrix,

$$
\mathscr{K}(x, \mathbf{P})=\left(\begin{array}{lllll}
0 & \omega K_{1} & \omega K_{2} & \omega K_{3} & E \\
-\omega K_{1} & 0 & \omega L_{3} & -\omega L_{2} & \omega R_{1} \\
-\omega K_{2} & -\omega L_{3} & 0 & \omega L_{1} & \omega R_{2} \\
-\omega K_{3} & \omega L_{2} & -\omega L_{1} & 0 & \omega R_{3} \\
-E & -\omega R_{1} & -\omega R_{2} & -\omega R_{3} & 0
\end{array}\right),
$$

whose elements transform as a skew-symmetric tensor on $M^{5}$, according to the rule

$\mathscr{K}_{(A B)}\left(x^{\prime}, \mathbf{P}^{\prime}\right)=\mathfrak{g}_{A}^{\cdot C} \cdot \mathfrak{g}_{B}^{\cdot D} \cdot \mathscr{K}_{(C D)}(x, \mathbf{P})$,

for all $\mathfrak{g} \in S O(1,4)$. Here $\mathfrak{g}_{A}^{\cdot B}=\eta_{A C}^{5} \mathfrak{g}_{\cdot D}^{C \cdot} \eta^{5 B D}$ are the matrix elements of the adjoint matrix $\overline{\mathfrak{g}}=\eta^{5} \mathfrak{g} \eta^{5}$. Thus, Eq. (19) can be written as $\mathscr{K}\left(x^{\prime}, \mathbf{P}^{\prime}\right)=\overline{\mathfrak{g}} \mathscr{K}(x, \mathbf{P}) \overline{\mathfrak{g}}^{T}$ or simpler, $\mathscr{K}^{\prime}=\overline{\mathfrak{g}} \mathscr{K} \overline{\mathfrak{g}}^{T}$.

Notice that all the conserved quantities carrying space indices $(i, j, \ldots)$ transform alike under rotations as $S O(3)$ vectors or tensors. Moreover, the condition $z^{i} \propto x^{i}$ fixes the same (common) three-dimensional basis $\left\{\boldsymbol{v}_{1}, \boldsymbol{v}_{2}, \boldsymbol{v}_{3}\right\}$ in both the Cartesian charts, of $M^{5}$ and $M$. This means that the $S O(3)$ symmetry is global [4] such that we may use the vector notation for the conserved quantities as well as for the local Cartesian coordinates on $M$. However, this basis must not be confused with that of the local frames on $M$ which are orthogonal in the sense of the dS geometry.

For studying the conserved quantities on the timelike geodesics we chose the chart $\{t, \rho, \theta, \phi\}$ taking the angular momentum along the third axis, $\mathbf{L}=L \boldsymbol{v}_{3}=(0,0, L)$, for restricting the motion in the equatorial plane, with $\theta=\frac{\pi}{2}$ and $\tilde{u}^{\theta}=0$. Then the non-vanishing conserved quantities can be written as

$$
\begin{aligned}
E= & \frac{m}{\tilde{\chi}^{2}} \tilde{u}^{t}, \\
L= & \frac{m \rho^{2}}{\tilde{\chi}^{2}} \tilde{u}^{\phi}, \\
K_{1}= & \frac{m}{\omega \tilde{\chi}^{2}}\left(\omega \rho \tilde{u}^{t} \cosh \omega t \cos \phi\right. \\
& \left.-\tilde{u}^{\rho} \sinh \omega t \cos \phi+\rho \tilde{u}^{\phi} \sinh \omega t \sin \phi\right), \\
K_{2}= & \frac{m}{\omega \tilde{\chi}^{2}}\left(\omega \rho \tilde{u}^{t} \cosh \omega t \sin \phi\right. \\
& \left.-\tilde{u}^{\rho} \sinh \omega t \sin \phi-\rho \tilde{u}^{\phi} \sinh \omega t \cos \phi\right), \\
R_{1}= & \frac{m}{\omega \tilde{\chi}^{2}}\left(\omega \rho \tilde{u}^{t} \sinh \omega t \cos \phi\right. \\
& \left.-\tilde{u}^{\rho} \cosh \omega t \cos \phi+\rho \tilde{u}^{\phi} \cosh \omega t \sin \phi\right), \\
R_{2}= & \frac{m}{\omega \tilde{\chi}^{2}}\left(\omega \rho \tilde{u}^{t} \sinh \omega t \sin \phi\right. \\
& \left.-\tilde{u}^{\rho} \cosh \omega t \sin \phi-\rho \tilde{u}^{\phi} \cosh \omega t \cos \phi\right),
\end{aligned}
$$

while $K_{3}=R_{3}=0$. Hereby we deduce the following obvious properties:

$\mathbf{K} \cdot \mathbf{L}=\mathbf{R} \cdot \mathbf{L}=0, \quad \mathbf{K} \wedge \mathbf{R}=-\frac{E}{\omega} \mathbf{L}$,

and verify the identities

$$
\begin{aligned}
& E^{2}-\omega^{2}\left(\mathbf{L}^{2}+\mathbf{R}^{2}-\mathbf{K}^{2}\right) \\
& =E^{2}-\omega^{2} \mathbf{L}^{2}-\mathbf{Q} \cdot \mathbf{P}=m^{2} \tilde{u}^{2}=m^{2},
\end{aligned}
$$


defining the principal invariant corresponding to the first Casimir operator of the $s o(1,4)$ algebra. In the flat limit, $\omega \rightarrow 0$, when $\mathbf{Q} \rightarrow \mathbf{P}$, this identity becomes just the usual mass-shell condition $p^{2}=m^{2}$ of special relativity $[6,10]$. We note that in the classical theory the second invariant of the $\operatorname{so}(1,4)$ algebra vanishes since there is no spin [6].

\section{Timelike geodesics}

In the case of the timelike geodesics we may exploit the identity $\tilde{u}^{2}=1$ and Eqs. (20) and (21) for obtaining the radial component

$\tilde{u}^{\rho}=\tilde{\chi}(\rho)^{2}\left[\frac{E^{2}}{m^{2}} \tilde{\chi}(\rho)^{2}-\frac{\omega^{2} L^{2}}{m^{2}}-\frac{L^{2}}{m^{2} \rho^{2}}-1\right]^{\frac{1}{2}}$,

which allows us to derive the following prime integrals:

$$
\begin{aligned}
\left(\frac{d \rho}{d t}\right)^{2}-\omega^{2} \rho^{2}+\frac{L^{2}}{E^{2} \rho^{2}} & =1-\frac{\omega^{2} L^{2}}{E^{2}}-\frac{m^{2}}{E^{2}}, \\
\frac{d \phi}{d t} & =\frac{L}{E \rho^{2}},
\end{aligned}
$$

which give the geodesic equations in the plane $\left(\boldsymbol{v}_{1}, \boldsymbol{v}_{2}\right)$ as

$$
\begin{aligned}
& \rho(t)=\left[-\kappa_{1}+\kappa_{2} \cosh 2 \omega\left(t-t_{0}\right)\right]^{\frac{1}{2}}, \\
& \phi(t)=\phi_{0}+\arctan \left[\sqrt{\frac{\kappa_{2}+\kappa_{1}}{\kappa_{2}-\kappa_{1}}} \tanh \omega\left(t-t_{0}\right)\right],
\end{aligned}
$$

where

$\kappa_{1}=\frac{E^{2}-m^{2}-\omega^{2} L^{2}}{2 \omega^{2} E^{2}}$,

$\kappa_{2}=\frac{1}{2 \omega^{2} E^{2}}\left[(E+m)^{2}+\omega^{2} L^{2}\right]^{\frac{1}{2}}\left[(E-m)^{2}+\omega^{2} L^{2}\right]^{\frac{1}{2}}$,

satisfying the identity

$\kappa_{2}^{2}-\kappa_{1}^{2}=\frac{L^{2}}{\omega^{2} E^{2}}$

Thus we solve the geodesic equation in terms of conserved quantities which give a physical meaning to the principal integration constants. The remaining ones, $t_{0}$ and $\phi_{0}$, determine only the initial position of the mobile and implicitly of its trajectory.

In this manner, we recover the well-known behavior of the timelike geodesic trajectory which is a hyperbola that for $\phi_{0}=0$ can be written easily in Cartesian space coordinates $(\rho, \phi) \rightarrow\left(\tilde{x}^{1}, \tilde{x}^{2}\right)$ of the plane $\left(\boldsymbol{v}_{1}, \boldsymbol{v}_{2}\right)$ as

$\tilde{x}^{1}(t)=\rho(t) \cos \phi(t)=\rho_{-} \cosh \omega\left(t-t_{0}\right)$,

$\tilde{x}^{2}(t)=\rho(t) \sin \phi(t)=\rho_{+} \sinh \omega\left(t-t_{0}\right)$, where

$\rho_{-}=\sqrt{\kappa_{2}-\kappa_{1}}, \quad \rho_{+}=\sqrt{\kappa_{2}+\kappa_{1}}$.

In the usual Cartesian coordinates these equations read

$x^{1}(t)=\frac{\rho_{-}}{\sqrt{1+\omega^{2} \rho(t)^{2}}} \cosh \omega\left(t-t_{0}\right)$,

$x^{2}(t)=\frac{\rho_{+}}{\sqrt{1+\omega^{2} \rho(t)^{2}}} \sinh \omega\left(t-t_{0}\right)$,

$x^{3}(t)=0$,

as they result from Eqs. (5), (31) and (32).

Now it remains to analyze the role of the conserved vectors $\mathbf{K}$ and $\mathbf{R}$, which depend on $E$ and $L$ as well as on $t_{0}$ and $\phi_{0}$. In the simpler case of $\phi_{0}=0$ their non-vanishing components read

$K_{1}=E \rho_{-} \cosh \omega t_{0}$,

$K_{2}=-E \rho_{+} \sinh \omega t_{0}$,

$R_{1}=E \rho_{-} \sinh \omega t_{0}$,

$R_{2}=-E \rho_{+} \cosh \omega t_{0}$

complying with the specific properties,

$K=|\mathbf{K}|=E\left[-\kappa_{1}+\kappa_{2} \cosh 2 \omega t_{0}\right]^{\frac{1}{2}}$,

$R=|\mathbf{R}|=E\left[\kappa_{1}+\kappa_{2} \cosh 2 \omega t_{0}\right]^{\frac{1}{2}}$,

$\mathbf{K} \cdot \mathbf{R}=E^{2} \kappa_{2} \sinh 2 \omega t_{0}$.

Moreover, the conserved momentum and its dual defined by Eq. (17) have the norms

$P=|\mathbf{P}|=\sqrt{2 \kappa_{2}} \omega E e^{\omega t_{0}}$,
$Q=|\mathbf{Q}|=\sqrt{2 \kappa_{2}} \omega E e^{-\omega t_{0}}$,

satisfying

$\mathbf{P} \cdot \mathbf{Q}=-2 \kappa_{1} \omega^{2} E^{2}$

Hereby we understand that the vector $\frac{\mathbf{K}}{E}$ indicates the position of the particle of mass $m$ at $t=0$ (Fig. 1) such that for $t_{0}=0$ this vector lays over the semi major axis being orthogonal on $\frac{\mathbf{R}}{E}$ (Fig. 2). It is remarkable that for any $t_{0}$ the vector $\mathbf{P}$ is oriented along the lower asymptote, while $\mathbf{Q}$ gives the direction of the upper one (as in Figs. 1 and 2). Thus the vector $\mathbf{Q}$, whose role in comoving charts was rather unclear [10], now gets a precise physical meaning.

All these properties are independent on the value of $\phi_{0}$ which gives only the rotation of the major axis in the plane $\left(\boldsymbol{v}_{1}, \boldsymbol{v}_{2}\right)$. Nevertheless, in the appendix we give the general form of all these conserved quantities calculated for an arbitrary $\phi_{0} \neq 0$. 


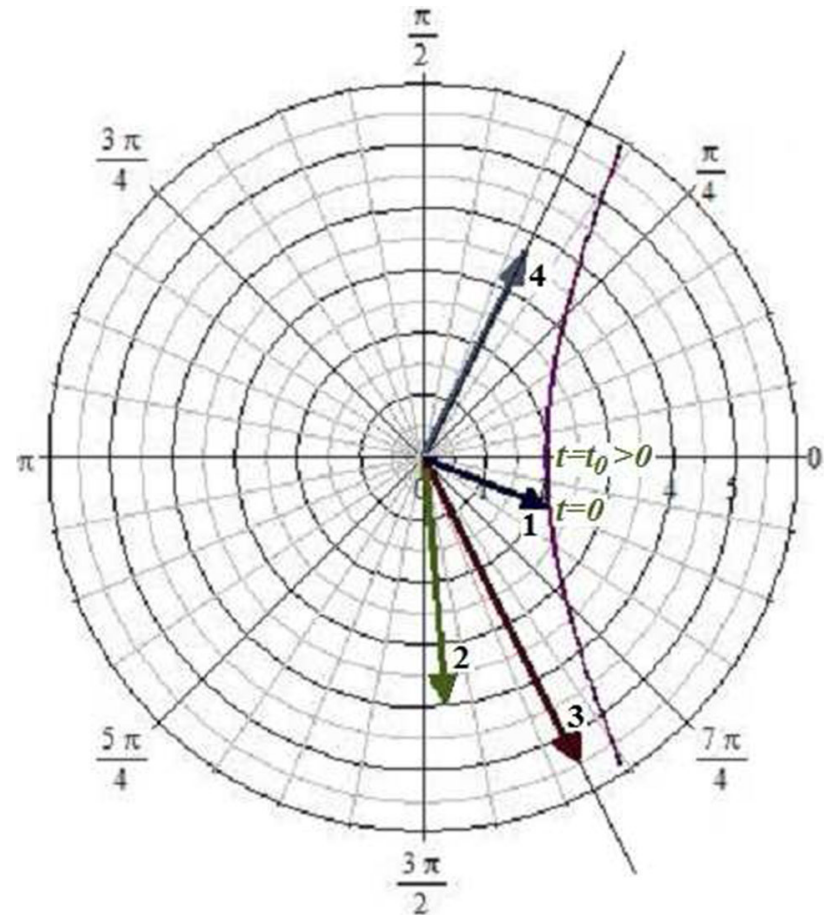

Fig. 1 de Sitter timelike geodesic with $\phi_{0}=0$ and $t_{0}>0$. The marked vectors are: (1) $\frac{\mathbf{K}}{E}$, (2) $\frac{\mathbf{R}}{E}$, (3) $-\frac{\mathbf{P}}{\omega E}$ and (4) $\frac{\mathbf{Q}}{\omega E}$

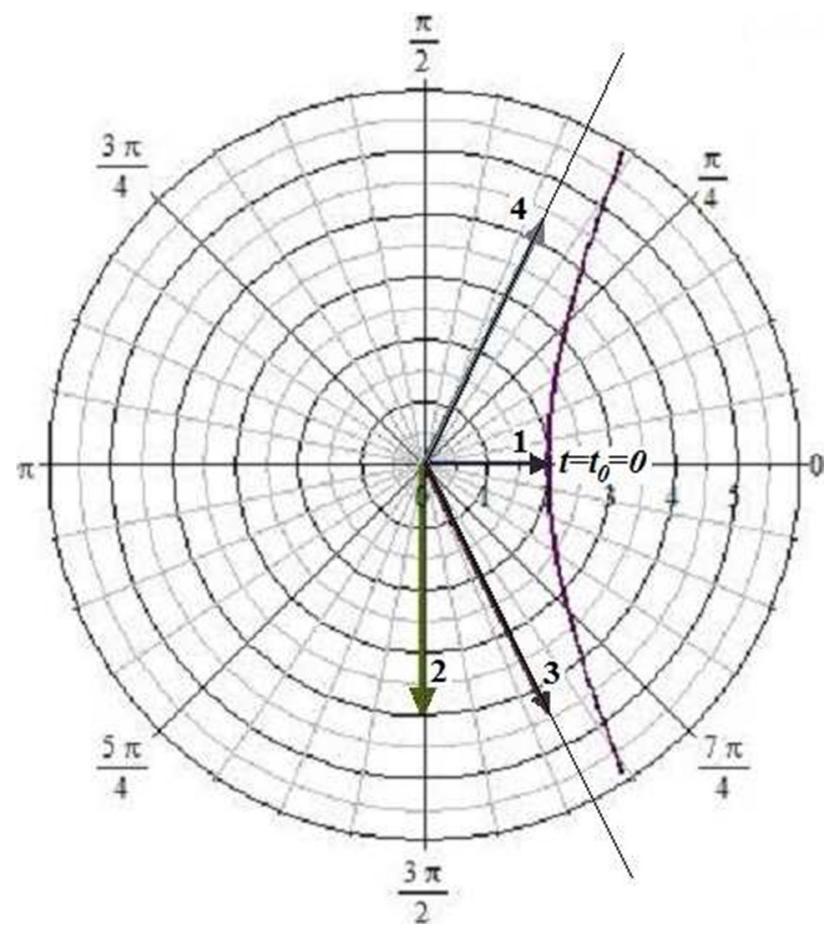

Fig. 2 de Sitter timelike geodesic with $\phi_{0}=0$ and $t_{0}=0$. The marked vectors are: (1) $\frac{\mathbf{K}}{E}$, (2) $\frac{\mathbf{R}}{E}$, (3) $-\frac{\mathbf{P}}{\omega E}$ and (4) $\frac{\mathbf{Q}}{\omega E}$

An important particular case is when the geodesic is passing through the origin since then the trajectory is rectilinear with $L=0$ and $\kappa_{-}=0$. Consequently, the vectors $\mathbf{P}$ and $\mathbf{Q}$ become parallel, having the norms

$$
P=e^{\omega t_{0}} \sqrt{E^{2}-m^{2}}, \quad Q=e^{-\omega t_{0}} \sqrt{E^{2}-m^{2}},
$$

resulting from Eqs. (49) and (50), and we obtain the geodesic equation

$x^{i}(t)=\frac{1}{\omega} \frac{P^{i} e^{-\omega t_{0}} \sinh \omega\left(t-t_{0}\right)}{\sqrt{E^{2}+P^{2} e^{-2 \omega t_{0}} \sinh ^{2} \omega\left(t-t_{0}\right)}}$,

and the four-velocity,

$$
\begin{aligned}
u^{0} & =\frac{1}{E m}\left[E^{2}+P^{2} e^{-\omega t_{0}} \sinh ^{2} \omega\left(t-t_{0}\right)\right], \\
u^{i} & =\frac{E}{m} \frac{P^{i} e^{-\omega t_{0}} \cosh \omega\left(t-t_{0}\right)}{\sqrt{E^{2}+P^{2} e^{-2 \omega t_{0}} \sinh ^{2} \omega\left(t-t_{0}\right)}} .
\end{aligned}
$$

Notice that if, in addition, we take $t_{0}=0$ then we have $\mathbf{K}=0, \mathbf{P}=\mathbf{Q}=-\omega \mathbf{R}$ and $P=Q=\sqrt{E^{2}-m^{2}}$, as in special relativity.

\section{Relativity}

Recently we have studied the relative geodesic motion on $\mathrm{dS}$ [10] and AdS manifolds [12], applying the Nachtmann method of boosting coordinates [3]. In the case of the AdS spacetimes we used static charts, while for the dS spacetimes we considered only comoving charts (i.e. the conformal Euclidean and de Sitter-Painlevé ones) where the geodesics are rectilinear [10]. Here we complete this study constructing the $\mathrm{dS}$ relativity in static charts by taking the results obtained previously in comoving charts, without revisiting the entire boosting method.

\subsection{Lorentzian isometries}

The problem of the relative motion is to find how an arbitrary geodesic trajectory and the corresponding conserved quantities can be measured by different observers. The local charts may play the role of inertial frames related through isometries. Each observer has its own proper frame $\{x\}$ in which he stays at rest in the origin on the world line along the vector field $\partial_{t}[10]$. Here we are interested in the inertial frames defined as proper frames of massive particles freely moving along geodesics. Then each mobile inertial frame can be labeled by the conserved quantities determining the geodesic of the carrier particle which stays at rest in its origin [10].

In what follows we consider two observers assuming that the first one, $O$, is fixed in the origin of his proper frame $\{x\}$ observing what happens in a mobile frame $\left\{x^{\prime}\right\}$ of the observer $O^{\prime}$, which is simultaneously the proper frame of $O^{\prime}$ and of a carrier particle of mass $m$ moving along a timelike geodesic with given parameters. This relativity does make 
sense only if we can compare the measurements of these observers imposing the synchronization condition of their clocks. This means that, at a given common initial time, the origins of these frames must coincide. However, this condition is restrictive since this forces the geodesic of the particle carrying the mobile frame to cross the origin of the fixed frame $O$. Consequently, its trajectory is rectilinear (with $\mathbf{L}=0$ ) in a given direction determined by its conserved momentum $\mathbf{P}$ as in Eq. (53).

The choice of the synchronization condition is a delicate point since the form of the isometry relating the fixed and mobile frames, called Lorentzian isometry, is strongly dependent on this condition. For this reason we use the same condition as in the case of the comoving frames [10] since then the Lorentzian isometry is generated by the same transformation of the $S O(1,4)$ group. Therefore, we set the synchronization condition at $t=t^{\prime}=0$ when $\mathbf{x}(0)=\mathbf{x}^{\prime}(0)=0$ such that the origins of both frames, $O$ and $O^{\prime}$, overlap the point $z_{o}=\left(0,0,0,0, \omega^{-1}\right)^{T} \in M^{5}$, which was the fixed point in constructing the dS manifold as the space of left cosets $S O(1,4) / L_{+}^{\uparrow}$ where the Lorentz group $L_{+}^{\uparrow}$ is the stable group of $z_{o}[10]$.

Under such circumstances, the synchronization condition is the same as in the case of the comoving charts and we can take over the $S O(1,4)$ transformation generating the Lorentzian isometry between the frames $O^{\prime}$ and $O$. This has the form [10]

$$
\begin{aligned}
\mathfrak{g}(\mathbf{P}) & =\exp \left(-i P^{i} \mathfrak{K}_{i} \frac{1}{P} \operatorname{arcsinh} \frac{P}{m}\right) \\
& =\left(\begin{array}{llllll}
\frac{E}{m} & \frac{P^{1}}{m} & \frac{P^{2}}{m} & \frac{P^{3}}{m} & 0 \\
\frac{P^{1}}{m} & 1+n_{p}^{12} v & n_{p}^{1} n_{p}^{2} v & n_{p}^{1} n_{p}^{3} v & 0 \\
\frac{P^{2}}{m} & n_{p}^{1} n_{p}^{2} v & 1+n_{p}^{2} v & n_{p}^{2} n_{p}^{3} v & 0 \\
\frac{P^{3}}{m} & n_{p}^{1} n_{p}^{3} v & n_{p}^{2} n_{p}^{3} v & 1+n_{p}^{32} v & 0 \\
0 & 0 & 0 & 0 & 1
\end{array}\right),
\end{aligned}
$$

where $\mathbf{n}_{P}=\frac{\mathbf{P}}{P}$ and $v=\left(\frac{E}{m}-1\right)$. The four-dimensional restriction of this transformation is a genuine Lorentz boost such that $\mathfrak{g}(\mathbf{P})^{-1}=\mathfrak{g}(-\mathbf{P})$ and $\mathfrak{g}(0)=\mathfrak{e}$. This transformation generates the Lorentzian isometry and transforms the conserved quantities according to Eq. (19).

The direct Lorentzian isometry, $x=\phi_{\mathfrak{g}(\mathbf{P})}\left(x^{\prime}\right)$, between the coordinates of the mobile and fixed frames, results from the system of equations $z(x)=\mathfrak{g}(\mathbf{P}) z\left(x^{\prime}\right)$ as

$$
\begin{aligned}
t\left(t^{\prime}, \mathbf{x}^{\prime}\right)= & \frac{1}{\omega} \operatorname{arctanh}\left(\frac{E}{m} \tanh \omega t^{\prime}\right. \\
& \left.+\frac{\omega}{m} \frac{\mathbf{x}^{\prime} \cdot \mathbf{P}}{\sqrt{1-\omega^{2}\left|\mathbf{x}^{\prime}\right|^{2}}} \operatorname{sech} \omega t^{\prime}\right),
\end{aligned}
$$

$$
\begin{aligned}
\mathbf{x}\left(t^{\prime}, \mathbf{x}^{\prime}\right)= & \mathbf{x}^{\prime}+\frac{\mathbf{P}}{m}\left(\frac{\mathbf{x}^{\prime} \cdot \mathbf{P}}{E+m}\right. \\
& \left.+\frac{1}{\omega} \sqrt{1-\omega^{2}\left|\mathbf{x}^{\prime}\right|^{2}} \sinh \omega t^{\prime}\right),
\end{aligned}
$$

while the inverse one has to be obtained by changing $x \leftrightarrow x^{\prime}$ and $\mathbf{P} \rightarrow-\mathbf{P}$. Obviously, in the limit of $\omega \rightarrow 0$ we recover the usual Lorentz transformations of special relativity.

We verify first that the geodesic trajectory of the carrier particle can be recovered from the parametric equations in $t^{\prime}$ obtained by substituting $\mathbf{x}^{\prime}=0$ in Eqs. (57) and (58). Then we obtain the trajectory of the origin $O^{\prime}$, denoted

$x_{*}^{i}(t)=\frac{P^{i} \sinh \omega t}{\omega \sqrt{E^{2}+P^{2} \sinh ^{2} \omega t}}$,

which is just Eq. (53) with $t_{0}=0$, corresponding to our initial condition $\mathbf{x}_{*}(0)=0$. The components of the four-velocity are those given by Eqs. (54) and (55) for $t_{0}=0$ when we have $E=m u_{*}^{0}(0)$ and $P^{i}=m u_{*}^{i}(0)$. This means that $E$ and $P^{i}$ are the components of the energy-momentum four-vector of the carrier particle when this is passing through the origin of the fixed frame.

This suggests us to consider as principal parameter the velocity of the carrier particle at $t=0$, defined usually as $\mathbf{V}=\frac{\mathbf{P}}{E}$. Then we may put the above formulas in forms closer to those of special relativity, eliminating the mass $m$ of the carrier particle. This can be done by changing the parametrization of $\mathfrak{g}(\mathbf{P})$, setting

$E=\gamma m, \quad \mathbf{P}=\gamma m \mathbf{V}, \quad \gamma=\frac{1}{\sqrt{1-V^{2}}}$,

such that we can rewrite

$\mathfrak{g}(\mathbf{P}) \rightarrow \mathfrak{g}(\mathbf{V})=\exp \left(-i V^{i} \mathfrak{K}_{i} \frac{1}{V} \operatorname{arctanh}(V)\right)$,

obtaining the new expression of the Lorentzian isometry

$$
\begin{aligned}
t\left(t^{\prime}, \mathbf{x}^{\prime}\right)= & \frac{1}{\omega} \operatorname{arctanh}\left(\gamma \tanh \omega t^{\prime}\right. \\
& \left.+\frac{\gamma \omega \mathbf{x}^{\prime} \cdot \mathbf{V}}{\sqrt{1-\omega^{2}\left|\mathbf{x}^{\prime}\right|^{2}}} \operatorname{sech} \omega t^{\prime}\right), \\
\mathbf{x}\left(t^{\prime}, \mathbf{x}^{\prime}\right)= & \mathbf{x}^{\prime}+\gamma \mathbf{V}\left(\mathbf{x}^{\prime} \cdot \mathbf{V} \frac{\gamma}{1+\gamma}\right. \\
& \left.+\frac{1}{\omega} \sqrt{1-\omega^{2}\left|\mathbf{x}^{\prime}\right|^{2}} \sinh \omega t^{\prime}\right),
\end{aligned}
$$

which may be used in applications.

The transformations $\mathfrak{g}(\mathbf{V})$, generating these isometries, transform simultaneously all the conserved quantities. If those of the mobile frame are encapsulated in the matrix 
$\mathscr{K}^{\prime}$ as in Eq. (18), then the corresponding ones measured in the fixed frame are the matrix elements of the matrix

$$
\mathscr{K}=\overline{\mathfrak{g}}(\mathbf{V}) \mathscr{K}^{\prime} \overline{\mathfrak{g}}(\mathbf{V})^{T}
$$

Thus we obtain the principal tools in studying the relative motion in static charts on dS spacetimes.

\subsection{Simple relativistic effects}

The principal feature of the Lorentzian isometries in $\mathrm{dS}$ static charts is that the domains of these mappings do not span the entire static charts involved in such transformations. Indeed, the condition $|\tanh z| \leq 1, \forall z \in \mathbb{R}$ indicates that Eq. (62) does make sense only in the domain $\mathscr{D}^{\prime}$ where the function

$B\left(\mathbf{x}^{\prime}\right)=\omega \frac{\mathbf{V} \cdot \mathbf{x}^{\prime}}{\chi\left(\mathbf{x}^{\prime}\right)}=\omega \frac{\mathbf{V} \cdot \mathbf{x}^{\prime}}{\sqrt{\left(1-\omega^{2}\left|\mathbf{x}^{\prime}\right|^{2}\right)}}$

satisfies

$-\frac{1}{\gamma} \cosh \omega t^{\prime}-\sinh \omega t^{\prime} \leq B\left(\mathbf{x}^{\prime}\right) \leq \frac{1}{\gamma} \cosh \omega t^{\prime}-\sinh \omega t^{\prime}$,

restricting thus the domain of the coordinates $\left(t^{\prime}, \mathbf{x}^{\prime}\right)$ of the mobile frame. This condition determines the field of view of the observer $O$ and guarantees that after this transformation we obtain well-defined Cartesian coordinates that satisfy the condition $\left|\mathbf{x}\left(t^{\prime}, \mathbf{x}^{\prime}\right)\right| \leq \frac{1}{\omega}$ imposed by the existence of the cosmological horizon. For the inverse Lorentzian isometry, we obtain a similar condition defining the domain $\mathscr{D}$ of this transformation.

It remains to investigate how this restriction works determining the domain $\mathscr{D}^{\prime}$. Assuming that the observation is along an arbitrary direction we denote $\alpha=\operatorname{angle}\left(\mathbf{x}^{\prime}, \mathbf{V}\right)$ such that we can write $B\left(\mathbf{x}^{\prime}\right)=\omega V \rho\left(\mathbf{x}^{\prime}\right) \cos \alpha$. Here $\rho$ is the radial coordinate defined by Eq. (5) that is free of any restriction, taking values in the domain $[0, \infty)$. Then the condition (66) restricts the observation at the points $\left(t^{\prime}, \mathbf{x}^{\prime}\right)$ which satisfy $\left|\rho\left(\mathbf{x}^{\prime}\right) \cos \alpha\right| \leq \rho_{\text {lim }}\left(V, t^{\prime}\right)$ where the function

$\rho_{\text {lim }}\left(V, t^{\prime}\right)=\frac{1}{\omega V}\left(\frac{1}{\gamma} \cosh \omega t^{\prime}-\operatorname{sign}\left(t^{\prime}\right) \sinh \omega t^{\prime}\right)$

is positively defined on the domain $\left[-t_{m}^{\prime}, t_{m}^{\prime}\right]$, vanishing for $t^{\prime}= \pm t_{m}^{\prime}$ where $t_{m}^{\prime}=\frac{1}{\omega} \operatorname{arctanh} \frac{1}{\gamma}$. Hence, we may conclude that Eq. (66) gives rise to non-trivial restrictions that seem to be specific for the static charts.

More interesting are the simple relativistic effects as the time dilation (observed in the twin paradox) and the Lorentz contraction. In general, these effects are quite complicated since they are strongly dependent on the position where the time and length are measured. Let us address this issue, assuming that the measurement is performed in the point $A$ of arbitrary position vector a, fixed rigidly to the mobile frame $O^{\prime}$. Then we may write the general relations

$$
\begin{gathered}
\delta t=\left.\frac{\partial t\left(t^{\prime}, \mathbf{x}^{\prime}\right)}{\partial t^{\prime}}\right|_{\mathbf{x}^{\prime}=\mathbf{a}} \delta t^{\prime}+\left.\frac{\partial t\left(t^{\prime}, \mathbf{x}^{\prime}\right)}{\partial x^{\prime} i}\right|_{\mathbf{x}^{\prime}=\mathbf{a}} \delta x^{\prime i}, \\
\delta x^{j}=\left.\frac{\partial x^{j}\left(t^{\prime}, \mathbf{x}^{\prime}\right)}{\partial t^{\prime}}\right|_{\mathbf{x}^{\prime}=\mathbf{a}} \delta t^{\prime}+\left.\frac{\partial x^{j}\left(t^{\prime}, \mathbf{x}^{\prime}\right)}{\partial x^{\prime i}}\right|_{\mathbf{x}^{\prime}=\mathbf{a}} \delta x^{\prime i},
\end{gathered}
$$

allowing us to relate among themselves the quantities $\delta t, \delta x^{j}$ and $\delta t^{\prime}, \delta x^{\prime} j$ measured by the observers $O$ and $O^{\prime}$.

We consider first a clock in $A$ indicating $\delta t^{\prime}$ without changing its position such that $\delta x^{\prime i}=0$. Then, after a little calculation, we obtain the time dilation observed by $O$, $\delta t(t)=\delta t^{\prime} \tilde{\gamma}(t)$, given by the function

$\tilde{\gamma}(t)=\gamma\left[1-B(\mathbf{a}) \sinh \omega t^{\prime}(t)\right] \frac{\cosh ^{2} \omega t}{\cosh ^{2} \omega t^{\prime}(t)}$,

which depends on the parameter $B$ (a) defined by Eq. (65) for $\mathbf{x}^{\prime}=\mathbf{a}$ and the function

$$
\begin{aligned}
t^{\prime}(t)= & \frac{1}{\omega} \operatorname{arctanh}\left[\frac{1}{\gamma\left(B(\mathbf{a})^{2}+1\right)}(\tanh \omega t\right. \\
& \left.\left.+B(\mathbf{a}) \sqrt{\gamma^{2} B(\mathbf{a})^{2}+\gamma^{2}-1+\operatorname{sech}^{2} \omega t}\right)\right],
\end{aligned}
$$

resulting after inverting Eq. (62) with $\mathbf{x}^{\prime}=\mathbf{a}$. Similarly but with the supplemental simultaneity condition $\delta t=0$ we derive the Lorentz contraction of an arbitrary $\delta \mathbf{x}^{\prime}$, which reads

$$
\begin{aligned}
\delta \mathbf{x}(t)= & \gamma \mathbf{n}_{V} \cdot \delta \mathbf{x}^{\prime}-B(\mathbf{a}) \mathbf{a} \cdot \delta \mathbf{x}^{\prime} \sinh \omega t^{\prime}(t) \\
& +\frac{\gamma^{2}-1}{\gamma} \frac{\cosh ^{2} \omega t^{\prime}(t)}{B(\mathbf{a}) \sinh \omega t^{\prime}(t)-1} \\
& \times\left[\mathbf{V} \cdot \delta \mathbf{x}^{\prime}+\frac{(\mathbf{V} \cdot \mathbf{a})\left(\mathbf{a} \cdot \delta \mathbf{x}^{\prime}\right)}{\chi(\mathbf{a})^{2}}\right],
\end{aligned}
$$

where $\mathbf{n}_{V}=\frac{\mathbf{v}}{V}$.

Thus we obtained the general formulas of the simple relativistic effects which hide a large phenomenology that cannot be exhaustively treated here because of the difficulties in studying analytically the general case of an arbitrary a. For this reason we restrict ourselves to the case of $\mathbf{a} \cdot \mathbf{V}=0$, assuming that $\delta \mathbf{x}^{\prime}$ is parallel with $\mathbf{V}$. Then, by taking $B(\mathbf{a})=0$ in Eqs. (70)-(72) we obtain

$\delta t=\delta t^{\prime} \tilde{\gamma}(t), \quad \delta x_{\|}=\delta x_{\|}^{\prime} \frac{1}{\tilde{\gamma}(t)}$

where the function $\tilde{\gamma}(t)$ takes the simple form

$$
\begin{aligned}
\tilde{\gamma}(t) & =\frac{\gamma}{\cosh ^{2} \omega t^{\prime}(t)-\gamma^{2} \sinh ^{2} \omega t^{\prime}(t)} \\
& =\gamma \cosh ^{2} \omega t-\frac{1}{\gamma} \sinh ^{2} \omega t
\end{aligned}
$$




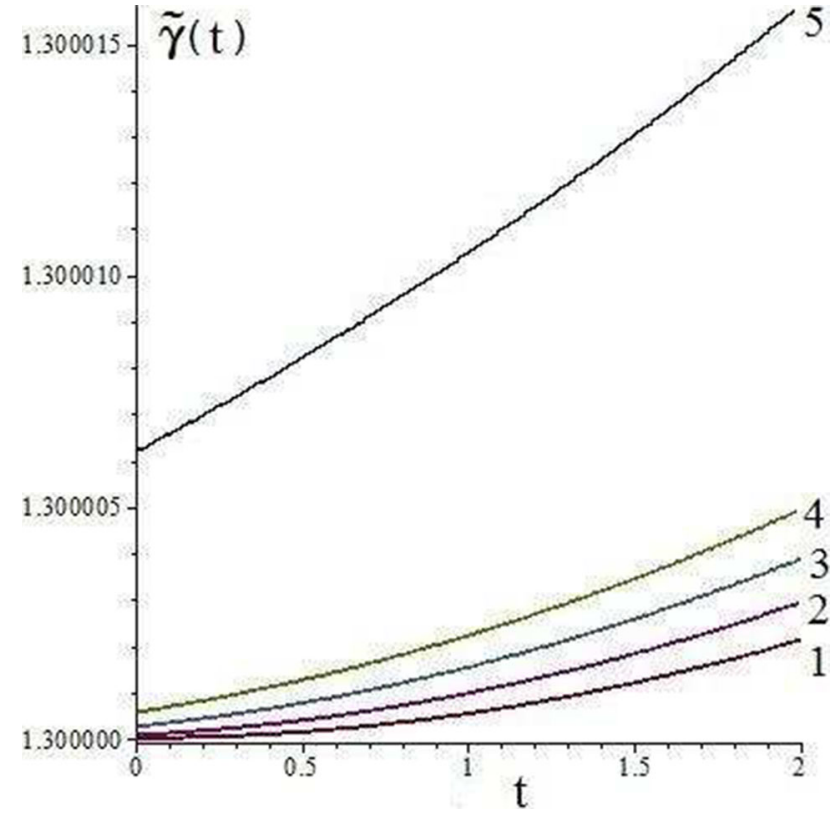

Fig. 3 Functions $\tilde{\gamma}(t)$ for $\omega=0.01, V=0.2$ and $a=0$ (1), $a=1$ (2), $a=2$ (3), $a=3$ (4) and $a=6$ (5)

since now $\gamma \tanh \omega t^{\prime}(t)=\tanh \omega t$. Hereby we recover again the well-known condition of the flat case, $\delta t \delta x_{\|}=\delta t^{\prime} \delta x_{\|}^{\prime}$, which also holds in the dS relativity in comoving charts [10].

The function $\tilde{\gamma}(t)$ is defined on the domain $(-\infty, \infty)$ taking values in the codomain $[\gamma, \infty)$. The time dilation observed by $O$ increases to infinity as

$\tilde{\gamma}(t) \sim \frac{1}{4}\left(\gamma-\frac{1}{\gamma}\right) e^{2 \omega t}$

when $t \rightarrow \infty$, since the observer $O$ sees how the clock in $O^{\prime}$ lats more and more such that $t$ tends to infinity when $t^{\prime}$ is approaching to $t_{m}$. Notice that the observer $O^{\prime}$ measures the same dilation of the time $t^{\prime}$ of a clock staying at rest in $O$.

In general, for the clocks situated in arbitrary space points the problem is much more complicated and cannot be solved without resorting to numerical method. As an example, we present in Fig. 3 the functions $\tilde{\gamma}(t)$ for different norms $a=|\mathbf{a}|$ of the position vector $\mathbf{a}$ oriented parallel with $\mathbf{V}$. Other interesting and attractive conjectures may be studied numerically starting with the above presented approach.

\section{Remark on the dS-AdS symmetry}

The Lorentzian isometry given by Eqs. (57) and (58) is related to the corresponding AdS isometry [12] through the transformation $\omega \rightarrow i \omega$. This is the effect of the well-known dS-AdS symmetry under this transformation, arising when one uses the same type of local charts in both these spacetimes. More- over, we must specify that this symmetry is general since the $\mathrm{dS}$ conserved quantities transform into AdS ones as

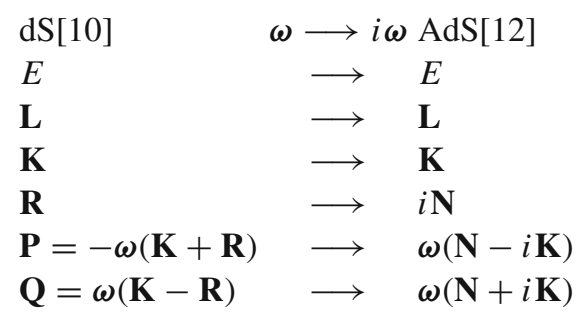

regardless of the local charts we consider. In other respects, this explains why in AdS spacetimes we do not have a realvalued conserved momentum.

Concluding we can say that the $\mathrm{dS}$ relativity in the conformal charts is closer to the Einstein special relativity having only rectilinear geodesics along the momentum directions, while in static charts the $\mathrm{dS}$ relativity is symmetric with the AdS one. Obviously, in the flat limit (when $\omega \rightarrow 0$ ) the $\mathrm{dS}$ and AdS relativity tend to the usual special relativity in Minkowski spacetime [6,9].

Finally, we note that this symmetry also holds at the level of the quantum theory where the quantum observables are conserved operators corresponding to the conserved quantities considered above, having the same physical meaning $[4,6]$. We remind the reader that in Ref. [4] the conserved observables of the covariant quantum fields of any spin on $\mathrm{dS}$ and AdS backgrounds are derived explicitly involving the dS-AdS symmetry. However, now it is premature to discuss how this symmetry may be extended to the quantum field theory, since even on dS spacetimes we have already the QED in Coulomb gauge [15]; on AdS spacetimes a similar theory has not yet been constructed.

Open Access This article is distributed under the terms of the Creative Commons Attribution 4.0 International License (http://creativecomm ons.org/licenses/by/4.0/), which permits unrestricted use, distribution, and reproduction in any medium, provided you give appropriate credit to the original author(s) and the source, provide a link to the Creative Commons license, and indicate if changes were made.

Funded by SCOAP $^{3}$.

\section{A Inverse problem}

There are situations when we know the integration constants $\kappa_{1}, \kappa_{2}, \phi_{0}$ and $t_{0}$ and we need to find the physical conserved quantities. Then from Eqs. (33) and (35) we deduce,

$$
\begin{aligned}
& E=\frac{m}{\sqrt{\left(\kappa_{1} \omega^{2}-1\right)^{2}-\kappa_{2}^{2} \omega^{4}}}, \\
& L=\frac{m \omega \sqrt{\kappa_{2}^{2}-\kappa_{1}^{2}}}{\sqrt{\left(\kappa_{1} \omega^{2}-1\right)^{2}-\kappa_{2}^{2} \omega^{4}}} .
\end{aligned}
$$


Furthermore, from Eqs. (22)-(25), calculated in aphelion, at $t=t_{0}$, where $\tilde{u}^{\rho}=0$ and $\tilde{u}^{t}$ and $\tilde{u}^{\phi}$ result from Eqs. (20) and (21), we derive the non-vanishing components

$$
\begin{aligned}
& K_{1}=E \rho_{-} \cos \phi_{0} \cosh \omega t_{0}+E \rho_{+} \sin \phi_{0} \sinh \omega t_{0}, \\
& K_{2}=E \rho_{-} \sin \phi_{0} \cosh \omega t_{0}-E \rho_{+} \cos \phi_{0} \sinh \omega t_{0} \\
& R_{1}=-E \rho_{-} \cos \phi_{0} \sinh \omega t_{0}+E \rho_{+} \sin \phi_{0} \cosh \omega t_{0}, \\
& R_{2}=-E \rho_{-} \sin \phi_{0} \sinh \omega t_{0}-E \rho_{+} \cos \phi_{0} \cosh \omega t_{0},
\end{aligned}
$$

while $K_{3}=N_{3}=0$. These components satisfy the properties (46)-(48).

\section{References}

1. W. de Sitter, On the curvature of space. Proc. Kon. Ned. Akad. Wet. 19 II, 1217 (1917)
2. S. Weinberg, Gravitation and Cosmology: Principles and Applications of the General Theory of relativity (Wiley, New York, 1972)

3. O. Nachtmann, Commun. Math. Phys. 6, 1 (1967)

4. I.I. Cotăescu, J. Phys. A Math. Gen. 33, 9177 (2000)

5. I.I. Cotăescu, Phys. Rev. D 65, 084008 (2002)

6. I.I. Cotăescu, GRG 43, 1639 (2011)

7. S. Cacciatori, V. Gorini, A. Kamenshchik, Ann. der Phys. 17, 728 (2008)

8. S. Cacciatori, V. Gorini, A. Kamenshchik, U. Moschella U, Class. Quantum Grav. 25, 075008 (2008)

9. I.I. Cotăescu, Phys. Rev. D 95, 104051 (2017)

10. I.I. Cotăescu, Eur. Phys. J. C 77, 485 (2017)

11. N.D. Birrel, P.C.W. Davies, Quantum Fields in Curved Space (Cambridge University Press, Cambridge, 1982)

12. I.I. Cotăescu, Phys. Rev. D 96, 044046 (2017)

13. E. van Beveren, G. Rupp, T.A. Rijken, C. Dullemond, Phys. Rev. D 27, 1527 (1983)

14. C. Dullemond, E. van Beveren, Phys. Rev. D 28, 1028 (1983)

15. I.I. Cotăescu, C. Crucean, Phys. Rev. D 87, 044016 (2013) 